\title{
Systemic Corticosteroid Therapy
}

National Cancer Institute

\section{Source}

National Cancer Institute. Systemic Corticosteroid Therapy. NCI Thesaurus. Code

C122080.

Treatment with corticosteroids via a delivery method that will affect the entire body (oral, intramuscular, intravenous). 